\title{
Frequency measurement under non-sinusoidal conditions
}

\author{
M. Mañana, J. A. Rodríguez, F. J. Sánchez, A. Ortiz, L. I. Eguíluz \\ Department of Electrical Engineering \\ E.T.S.I.I.T., University of Cantabria \\ Avda. Los Castros s/n, 39005 Santander (Spain) \\ phone:+34 942201378, fax:+34 942201385, e-mail: mananam@unican.es
}

\begin{abstract}
Frequency measurement is an important issue in electrical engineering. Electric power systems have become increasing complex over the last decade. The use of distributed generation, the connection of non-linear loads and the presence of unexpected system faults are the main causes of frequency variations. In addition, power quality includes frequency as an important index.

From a hardware instrumentation point of view, frequency measurement has different requirements:
\end{abstract}

i.) Large power systems have slow frequency variation due to the high inertia of the overall network. This kind of problem requires frequency measuring methods capable of detecting small and also slow frequency variations.

ii.) Small power systems can have frequency variation due to their reduced short-circuit power capacity. This type of problem requires fast methods with the capacity to detect large frequency variations.

There are different groups of methods intended for frequency measurement. The methods can be compared in terms of computation and dynamic response, especially when the main voltage is disturbed.

This research work focuses on frequency measurement under non-sinusoidal conditions. The paper studies the behaviour of a modified version of Sezi's method and its hardware implementation using a microcontroller. This system can be used for frequency measurement or as a synchronized sampling source in harmonic measurement (e.g. EN 61000-4-7)

The fast time response of the system enables it to be used in almost all kinds of application: small and slow frequency variations; frequency triggering in power system protection and power quality index characterisation.

\section{Key words}

frequency meters, power quality, non-sinusoidal conditions, instrumentation.

\section{Introduction}

Most power system experts do not consider frequency variation as an important topic because it is rather difficult to find large frequency variation in continental power networks. However, from the point of view of the instrumentation design, some instrumentation devices used to register power quality disturbances exhibit a great sensitivity to small frequency variations. For instance, the European standard EN 61000-4-7 [1] defines the characteristics of an instrumentation device used for harmonic distortion measurement based on analog to digital conversion. This device includes a phase locked loop in order to adjust the sampling frequency dynamically but does not include any comment about the behaviour of the method used to compute the fundamental frequency.

The paper includes a review of the behaviour of frequency measurement under non-sinusoidal conditions from two points of view:

- Frequency measurement for power quality assessment according to EN 50160 [2]. This document defines the characteristics of the supplied voltage: frequency, magnitude variations, unbalance, harmonics, surges, etc.

- Frequency measurement as a reference for sampling control in analog to digital conversion. (e.g. EN 61000-4-7)

From the perspective of the type of algorithm used for frequency computation, there are several possibilities:

- Adaptive Kalman filtering methods.

- Zero-cross detection methods.

- Discrete Fourier Transform methods.

- FIR/IIR iterative methods.

- Other methods.

In order to evaluate the differences between the different methods, some algorithms have been implemented using a high performance microcontroller PIC 18F452 [3] from Microchip. The designed hardware includes a RS232 connection in order to send the computed data to a PC. Figure 1 shows the block diagram of the proposed system.

An iterative algorithm based on FIR filter has showed a good behaviour under non-sinusoidal conditions [4]. The method is not dependent upon zero-crossing of the measured voltage or current. In addition it can compute 


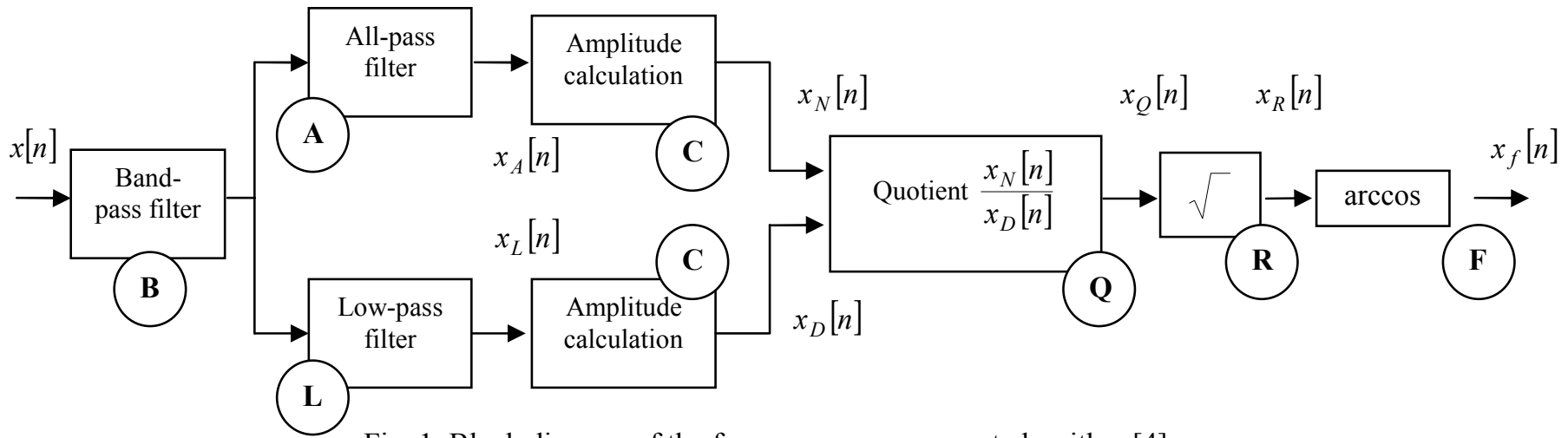

Fig. 1. Block diagram of the frequency measurement algorithm [4].

the frequency after every sample. In addition, the method only needs a 3 -cycles signal-window.

The accuracy of the computed frequency is better than 10 $\mathrm{mHz}$ for a nominal frequency of $50 \mathrm{~Hz}$.

This frequency meter is basically insensitive to steady state disturbances like harmonics or low frequency amplitude variations so it can be used as a reference method in order to obtain an accurate sampling frequency in harmonic meters

\section{Frequency measurement under non- sinusoidal conditions}

The above methods can be included in one of three main principles that support frequency measurement [5]:

- $\quad$ Period estimation based on the measurement of time interval between zero-crossing [6].

- $\quad$ DFT based method, which uses interpolation in frequency domain $[7,8]$.

- Orthogonal phasor decomposition with local determination of rotation speed [9-12].

The behaviour of each group of methods under non sinusoidal conditions depends on the kind of disturbance. In general, zeros-crossing methods can be used with harmonic distortion, especially with high-order harmonics.

DFT methods have to be implemented as block algorithms so this kind of procedures needs from two to eight cycles to compute frequency.

Orthogonal phasor decomposition, like the zero-crossing method, belongs to the group of iterative filter methods.

All methods are sensitive to transient distortion such as sags, swells and impulses. On the other hand, they are rather insensitive to white noise and harmonic periodic distortion.

\section{Frequency algorithm}

The proposed algorithm is based on Sezi's method [4]. This algorithm uses phase angle and amplitude information in order to compute frequency. This method avoids waiting between zero-crossing. Figure 1 shows the block diagram of Sezi's method with the stages involved.

The sampled data $x[n]$ is first filtered by a band-pass FIR filter B. The main aim of this block is to filter all harmonics, subharmonics and inter-harmonic distortion. This bandpass filter $\mathrm{B}$ has been designed with the following restrictions:

Equiripple FIR bandpass filter of order 40

Sampling frequency $1000 \mathrm{~Hz}$

Fstop $_{1}=10 \mathrm{~Hz} ;$ Fpass $_{1}=30 \mathrm{~Hz}$

Fpass $_{\mathrm{h}}=80 \mathrm{~Hz}$; Fstop $\mathrm{h}=120 \mathrm{~Hz}$

Amplitude $_{\text {pass }}=1$

Amplitude $_{\text {stopl }}=60 ;$ Amplitude $_{\text {stoph }}=80$

The filter coefficients are included in Table I.

TABLE I. Bandpass filter B coefficients.

\begin{tabular}{|l|l|l|l|}
\hline Coef. & Value & Coef. & Value \\
\hline 1 & 0.007749520989041 & 21 & 0.123420397846289 \\
2 & 0.012535189342240 & 22 & 0.115871771716403 \\
3 & 0.019230726379304 & 23 & 0.094319125134788 \\
4 & 0.024578269196053 & 24 & 0.061865040318399 \\
5 & 0.026195484608224 & 25 & 0.023205602586402 \\
6 & 0.021918288148958 & 26 & -0.016101012847003 \\
7 & 0.010529708044245 & 27 & -0.050489670521057 \\
8 & -0.007644015196679 & 28 & -0.075333780176352 \\
9 & -0.030509573299857 & 29 & -0.087736170069564 \\
10 & -0.054396150203906 & 30 & -0.086999488341449 \\
11 & -0.074734365352290 & 31 & -0.074734365352290 \\
12 & -0.086999488341449 & 32 & -0.054396150203906 \\
13 & -0.087736170069564 & 33 & -0.030509573299857 \\
14 & -0.075333780176352 & 34 & -0.007644015196679 \\
15 & -0.050489670521057 & 35 & 0.010529708044245 \\
16 & -0.016101012847003 & 36 & 0.021918288148958 \\
17 & 0.023205602586402 & 37 & 0.026195484608224 \\
18 & 0.061865040318399 & 38 & 0.024578269196053 \\
19 & 0.094319125134788 & 39 & 0.019230726379304 \\
20 & 0.115871771716403 & 40 & 0.012535189342240 \\
& & 41 & 0.007749520989041 \\
\hline
\end{tabular}


Figure 2 shows the amplitude versus frequency response of the bandpass filter.

Amplitude (dB)

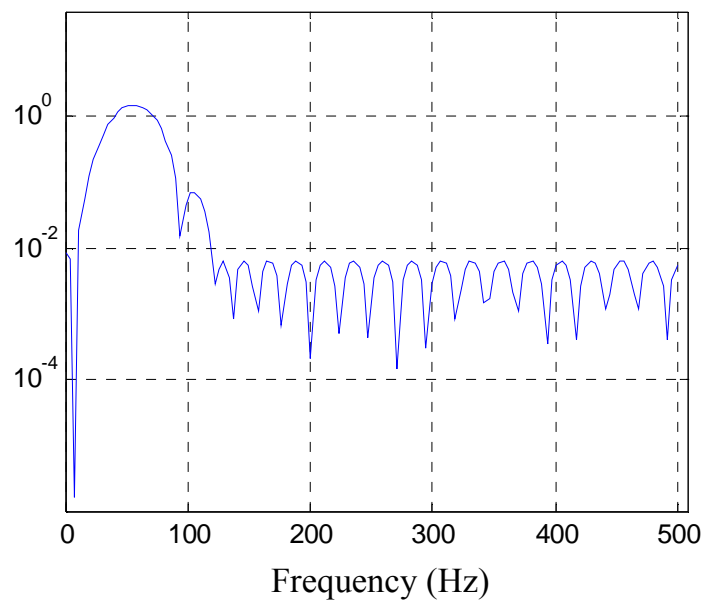

Fig. 2. Amplitude response of bandpass filter B.

The output of the bandpass filter B is connected in two different ways. The first one is an all-pass filter whereas the second is a low-pass filter. Equations (1) and (2) show the frequency transfer function of both filters,

$$
\begin{gathered}
A(f)=1 \\
L(f)=1+\cos (5 \Omega) \\
\Omega=2 \pi\left(\frac{f}{f_{s}}\right)
\end{gathered}
$$

where

$$
\begin{array}{ll}
\Omega & \text { is the normalized frequency, } \\
f & \text { actual frequency } \\
f_{s} & \text { sampling frequency }
\end{array}
$$

The $\mathrm{Z}$ domain transfer function of filters $\mathrm{A}$ and $\mathrm{L}$ are

$$
\begin{gathered}
H_{A}(z)=z^{-5} \\
H_{L}(z)=0.5+z^{-5}+0.5 z^{-10}
\end{gathered}
$$

Figure 3 shows the amplitude versus frequency response of filters $\mathrm{A}$ and $\mathrm{L}$.

The outputs of filters $\mathrm{A}$ and $\mathrm{L}$ pass through a block $\mathrm{C}$ that computes the amplitude of the signals $x_{A}[n]$ and $x_{L}[n]$. In fact, block $C$ obtains a value proportional to the square of the signal amplitude. The proposed C block [13] differs from original Sezi's in some aspects: i) It is a IIR filter and ii) It has a reduced computational load.

The difference equation of this filter is,

$$
y_{C}[n]=y_{C}[n-1]+0.1\left(x_{C}^{2}[n]-x_{C}^{2}[n-10]\right)
$$

The block Q computes the quotient of the signals $x_{N}[n]$ and $x_{D}[n]$. The output of block $\mathrm{Q}$ is root squared in order to obtain $x_{R}[n]$. The amplitude frequency response of signal $x_{R}[n]$ is,

$$
x_{R}[n]=[1+\cos (5 \Omega)]
$$

The last block $\mathrm{F}$ performs the inverse of cosine function in order to obtain $\Omega$ from equation (7),

$$
\Omega=\frac{1}{5} \arccos \left(x_{R}[n]-1\right)
$$

The actual frequency $\mathrm{f}$ can be directly obtained from expression (8),

$$
f=\left(\frac{f_{s}}{2 \pi}\right) \Omega
$$

Another advantage of Sezi's method is that frequency dependent errors are eliminated because all the computations are done using the same numerical process.

Amplitude

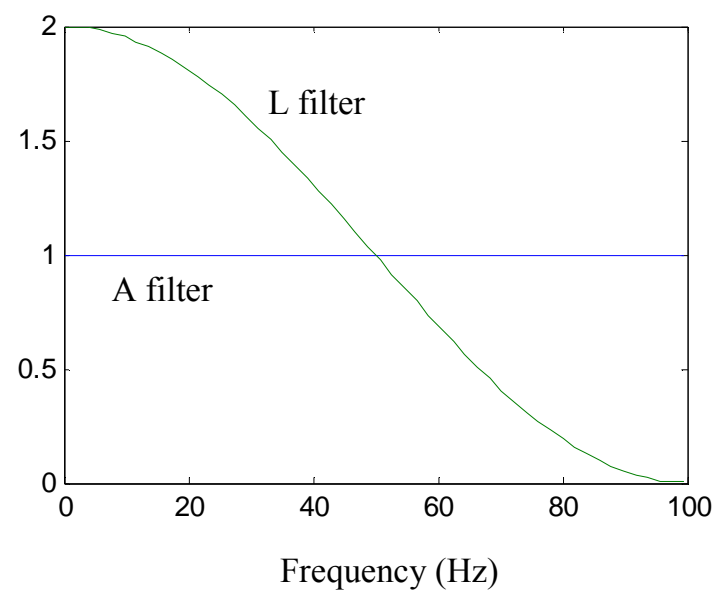

Fig. 3. Amplitude versus frequency response of filters $\mathrm{A}$ and $\mathrm{L}$.

\section{Instrumentation architecture}

The algorithm has been implemented using a Microchip ${ }^{\circledR}$ microcontroller PIC 18F452 [3]. This microcontroller is a high performance RISC device with $32 \mathrm{kB}$ (16.384 single-word instructions) on-chip program memory and a computation speed up to 10 MIPS with internal hardware multiplier. In spite of the fact that it has a lot of peripherals, it is important to highlight its eight 10 bits analog to digital converter. 
Figure 4 shows the instrumentation basic block diagram.

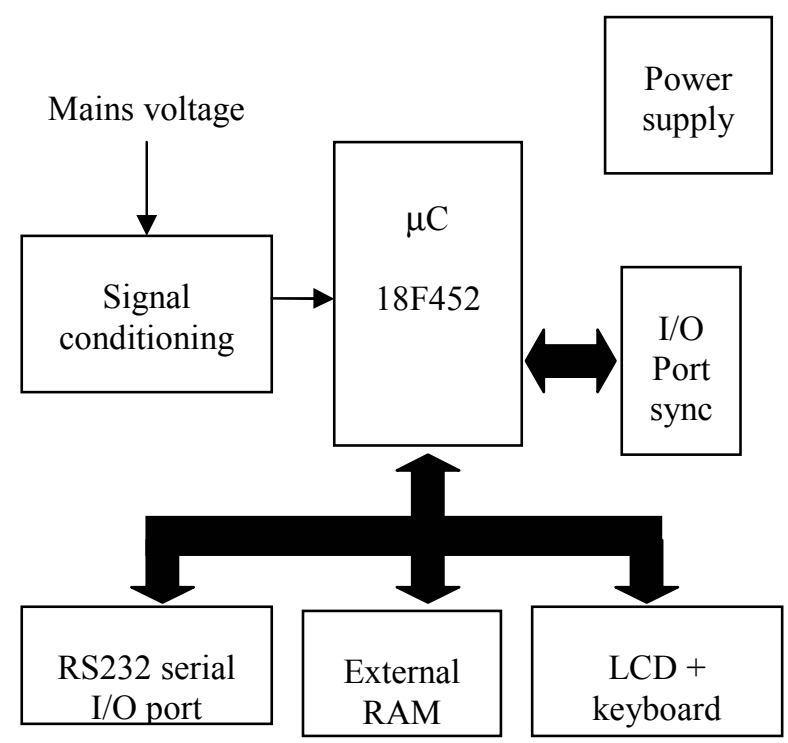

Fig. 4. Instrumentation block diagram.

The hardware designed has some advantages from the point of view of the connexion with other instrumentation:

- It is extremely simple and cheaper. In fact, all the design can be reduced to the microcontroller without the LCD, keyboard and I/O serial port.

- The synchronization I/O port can be used as a trigger source for other power quality instruments or protection devices.

\section{Test results}

The implemented hardware has been putting to the test with an arbitrary programmable power supply in order to know the accuracy and time response.

This programmable three phase source HP6834B [14] allows arbitrary waveforms to be generated with a maximum power of $1500 \mathrm{VA}$ in every phase or $4500 \mathrm{VA}$ in the case of one phase generation. The power supply is controlled using a PC and a GPIB link.

This frequency meter has been tested with two kind of signals: i) sinusoidal and ii) non-sinusoidal.

Table II summarizes statistical results obtained from sinusoidal signals with frequencies varying from 46 to 54 $\mathrm{Hz}$ in steady state.

TABLE II. Statistical values obtained from test signals with frequencies varying from 46 to $54 \mathrm{~Hz}$.

\begin{tabular}{|l|l|l|l|l|l|}
\hline frequency & 46 & 48 & 50 & 52 & 54 \\
\hline Min & 45.9369 & 47.9426 & 49.9257 & 51.9039 & 53.8788 \\
\hline Mean & 46.0002 & 48.0002 & 50.0005 & 52.0003 & 54.0001 \\
\hline Max & 46.0679 & 48.0606 & 50.0749 & 52.0927 & 54.1252 \\
\hline Std & 0.0386 & 0.0383 & 0.0469 & 0.0599 & 0.0794 \\
\hline
\end{tabular}

The time needed to reach the steady state is over three cycles but can be reduced if filter with shorter order are used.

$\mathrm{f}(\mathrm{Hz})$

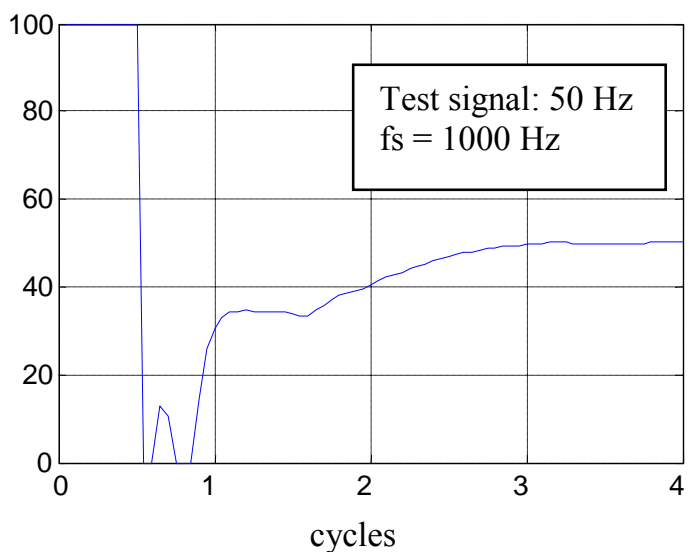

Fig 5. Time evolution of computed frequency during system initialization.

The transient response showed in Figure 5 also appears when the main signal is disturbed with some disturbances like sags, swell and impulses. Figure 6 shows the transient response during a $50 \%$ sag.

Finally, the system was tested using a non-sinusoidal signal disturbed with harmonics of orders three $(30 \%)$ and five $(20 \%)$. Figure 7 shows the steady state response.

$\mathrm{f}(\mathrm{Hz})$

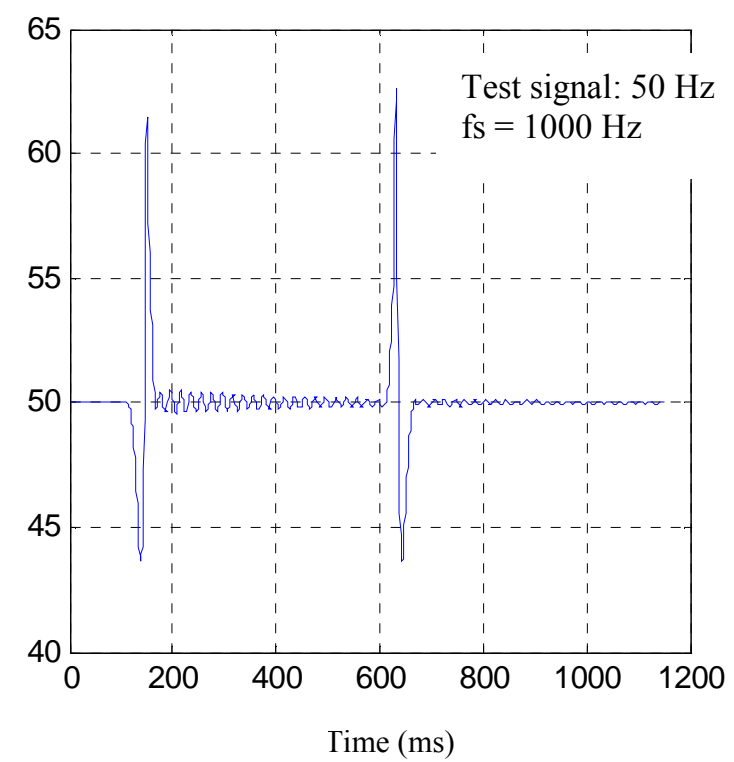

Fig. 6. Transient response of computed frequency during a $50 \%$ voltage sag. 


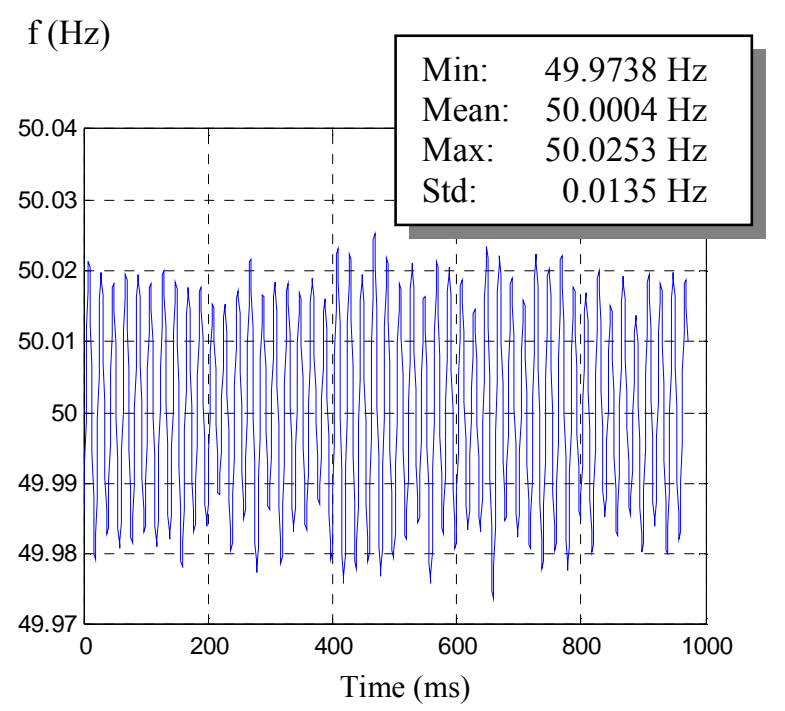

Fig. 7. System response with a $50 \mathrm{~Hz}$ signal disturbed with harmonics of orders three and five.

\section{Conclusions}

It has been probed that the modified Sezi's method has a good response under both sinusoidal and non-sinusoidal conditions.

The modification of the amplitude computation block $\mathrm{C}$ reduces the computational requirements. The overall system has been implemented in a real minimum hardware system based on a microcontroller.

One of the major advantages of this method is that this algorithm calculates the system frequency after every data sampling with a reduced computational load.

The initialization time is over three periods of $50 \mathrm{~Hz}(60$ $\mathrm{ms})$.

The system exhibits some instability when the amplitude changes suddenly, as in sags and transients. One way to solve this problem is watching the calculate frequency. If the frequency is out the range $[40,60] \mathrm{Hz}$, then the computed frequency is not valid and a warning digital output is set on.

\section{References}

[1] CENELEC EN 61000-4-7. Electromagnetic compatibility (EMC) -- Part 4-7: Testing and measurement techniques - General guide on harmonics and interharmonics measurements and instrumentation, for power supply systems and equipment connected thereto. 1997.

[2] CENELEC EN 50160.Voltage characteristics of electricity supplied by public distribution systems. 1999.

[3] Microchip PIC 18F452. Datasheet.

[4] Sezi, T., "A New Method for Measuring Power System Frequency". IEEE Transmission and Distribution Conference. Vol. 1, p 400-405. 11-16 April 1999.
[5] Asnin, L.; Backmutsky, V.; Gankin, M.; "DSP methods for dynamic estimation of frequency and magnitude parameters in power systems transients". IEEE Porto Power Tech Conference. 2001.

[6] Vainio, O.; Ovaska, S.; "Digital Filtering for Robust $50 / 60 \mathrm{~Hz}$ Zero-Crossing Detectors". IEEE Transactions on Instrumentation and Measurement, Vol. 45, No. 2, p 426-430. April 1996.

[7] Yang, J.; Liu, C.; "A Precise Calculation of Power System Frequency". IEEE Transactions on Power Delivery. Vol. 16, No. 3. p 361-366. July 2001.

[8] Agrez, D.; "Frequency Estimation of the NonStationary Signals Using Interpolated DFT". IEEE Instrumentation and Measurement Technology Conference. p- 925-930. 2002.

[9] Kezunovic, M.; Spasojevic, P.; Perunicic, B., "New Digital Signal Processing Algorithms for Frequency Deviation Measurement". IEEE Transactions on Power Delivery, Vol. 7, No. 3, p 1563-1573. July 1992.

[10] Szafran, J.; Rebizant, W.; "Power System Frequency Estimation". IEE Proc.-Gener. Transm. Distrib., Vol 145, No 5, September 1998.

[11] Lin, T.; Tsuji, M.; Yamada, E.; "A Wavelet Approach to Real Time Estimation of Power System Frequency". Proceedings of the $40^{\text {th }}$ SICE Annual Conference. 113 C-1. p 58-65. July 25-27. Nagoya. 2001.

[12] Hocaoglu, A.; Devaney, M.; "A New Quadratic Form Based Frequency Measurement Algorithm". IEEE Instrumentation and Measurement Technology Conference. p 1065-1070. Brussels. June 4-6. 1996.

[13] Mañana, M.; "Contributions to the representation, detection and classification of disturbances in power system" PhD thesis. (in Spanish). University of Cantabria. 2000.

[14] HP 6834B. User’s Guide. 1996. 